\title{
Noninvasive Identification of Core Gene Biomarkers in Patients with Acute Kidney Transplant Rejection
}

Huijia Zhao

Wuhan University Zhongnan Hospital

Ling Li

Wuhan University Zhongnan Hospital

Qi-fa Ye ( $\nabla$ yqf_china@163.com )

Wuhan University Zhongnan Hospital https://orcid.org/0000-0002-2797-4804

Research article

Keywords: Acute rejection, bioinformatical analysis, immune response, ncRNA transport, oxidative stress

Posted Date: June 12th, 2020

DOI: https://doi.org/10.21203/rs.3.rs-34209/v1

License: (c) (i) This work is licensed under a Creative Commons Attribution 4.0 International License.

Read Full License 


\section{Abstract}

Background: Acute rejection (AR) is one of common and critical complications after kidney transplantation, which gives rise to an increasing of allograft loss and even death. However, the potential mechanisms of AR remain incompletely understood at present. This study aimed to reveal the underlying mechanisms and identify core biomarkers of AR.

Methods: The AR gene expression profile GSE1563 involving 6 renal transplant patients undergoing AR and 9 patients with well-functioning transplant with no clinical evidence of rejection was selected to analyze the differentially expressed genes (DEGs) from purified RNA of peripheral blood lymphocytes. DAVID was used to carry out Gene ontology (GO) and the Kyoto Encyclopedia of Genes and Genomes (KEGG) pathway analyses. A protein-protein interaction (PPI) network was built to display the interactions among these DEGs. To get further reliable significant genes and mechanisms, gene set enrichment analysis (GSEA) was applied to evaluate the hub gene analyzing via PPI network.

Results: A total of 347 DEGs were captured, including 301 upregulated genes and 46 downregulated genes. Go and KEGG pathway analysis showed the DEGs were particularly enriched in immune response, inflammatory response to antigenic stimulus, RNA transport and protein stabilization. The PPI network indicated 3 modules were also mainly involved in immune response and RNA transport. 18 hub genes were selected in PPI network, among which there were 6 core genes evaluated by DAVID. By the way of GSEA, Oxidative stress was another potential mechanism besides immunity and ncRNA transport.

Conclusion: Our analysis uncovered the immune response including humoral and cellular immunity, ncRNA transport as well as oxidative stress was the major mechanisms of AR associated respectively with MAPK8, CCL5, HMGB1, NCBP2, XPO1 and APP, which could be novel noninvasive biomarkers in peripheral blood for early diagnosis and could be helpful to the treatment of AR.

\section{Introduction}

Kidney transplantation is a crucial therapy for patients with end-stage kidney disease (ESKD), as it enhances the quality of patients' life and reduces mortality compared with dialysis ${ }^{[1]}$. Due to the usage of advanced immunosuppressive medications and the improvement of organ procurement methods, the 5year deceased donor kidney allograft survival rates have increased up to $72.4 \%{ }^{[2]}$. However, the risk of allograft loss, especially acute graft rejection remain a major hurdle to overcome ${ }^{[3]}$. In a study of a consecutive series of 319 cases receiving deceased kidney transplantations over a 7 year period, $31 \%$ experience a single early acute rejection ${ }^{[4]}$. The potential mechanisms of acute rejection (AR) following kidney transplantation remain unclear. In this context, it is elementary to promote our understanding between immune system and the transplanted organ to immune and non-immune mechanisms of injury.

Generally, the gold standard for diagnosis of AR is histology obtained via needle biopsy, whereas this technique is infrequently used for surveillance as a result of cost, potential complications, patient 
discomfort and inconvenience ${ }^{[5]}$. Furthermore, other laboratory examinations involving serum creatinine, proteinuria and donor-specific antibodies as well as some non-invasive tests like renal ultrasound with doppler for renal arterial and venous indices also can help to evaluate the condition of AR. However, these methods for AR diagnosis display poor specificity and efficiency, which are difficult for us to gain accurate diagnosis and proper treatment early, so that some patients with AR lost the best chance for therapy, thereby causing graft dysfunction and even improving the death risk ${ }^{[6]}$. Hence, the identification of specific and sensitive biomarkers is essential to assist us accurate diagnosis and treatment of AR as soon as possible, especially noninvasive biomarkers.

High-throughput gene microarray and bioinformatic analysis have enabled us to screen molecular markers among healthy individuals and patients, which provides novel perceptions into diseases at multiple levels ranging from the variation of copy number at the genome level to gene expression at transcriptome and protein level, and even epigenetic alterations ${ }^{[7]}$. However, the application of microarrays in clinic is limited due to overwhelming number of genes identified by gene profiling, shortage of repeatable and independent validation and complex statistical analyses ${ }^{[8]}$. Moreover, the majority of the microarrays are grounded on the genes in tissues which is hard to verify and test except by invasive methods ${ }^{[9]}$. As a result, for the sake of put these expression profiles into clinical applications as quickly as possible, it is vital to confirm a suitable amount of serum genes and develop an appropriate and perfect way which can be worked out by routine assay.

In this study, the gene expression profile of GSE1563 was downloaded from the Gene Expression Omnibus (GEO, http://www.ncbi.nIm.nih.gov/geo/) which is an online public collection database for microarray data and used GEO2R online software to thoroughly identify differentially expressed genes (DEGs) between kidney transplantation patients with and without AR. Then, we analyzed gene ontology (GO) including biological processes (BP), molecular function (MF), cellular component (CC), and KEGG pathway of the DEGs. Furthermore, we constructed protein-protein interaction (PPI) network of these DEGs and picked up the top 12 hub genes with a high degree of connectivity. Subsequently, we carried out three modules and confirmed their enriched pathway. After the analysis of hub genes, we sustained the enrichment of hub genes to find the pivotal mechanism of AR according to Gene Set Enrichment Analysis (GSEA).

\section{Materials And Methods}

2.1 Microarray Data we downloaded the gene expression profile of GSE1563 from the National Center for Biotechnology Information (NCBI) GEO database, a free and publicly available database ${ }^{[10]}$. We selected a total of 15 samples of purified RNA of peripheral blood lymphocytes (PBL), involving 6 renal transplant patients undergoing AR and 9 patients with well-functioning transplant with no clinical evidence of rejection, which was based on GPL8300 platform [HG_U95Av2] Affymetrix Human Genome U95 Version 2 Array by Flechner SM et al. We also obtained the Series Matrix File of GSE1563 from the GEO database in pubmed. Inclusion criteria of AR group for this microarray was according to Banff criteria and confirmed 
by response to anti-rejection therapy ${ }^{[11]}$. Transplant patients with CMV or other infections in clinical and laboratory evidence were excluded. Immunosuppression medications contained a calcineurin inhibitor or sirolimus, with mycophenolate mofetil and steroids. Additionally, patients with no rejection were in line with more than one-year post transplant in patients with good transplant function and normal histology. Genes expression profile was evaluated by Affymetrix HG-U95Av2 GeneChip arrays via total RNA extracted from all samples.

2.2 Screen Genes of Differential Expression The screening of DEGs between AR and no rejection samples was performed by using GEO2R((http://www.ncbi.nlm.nih.gov/geo/geo2r), which is a online analysis tool for the GEO database according to $\mathrm{R}$ language. Compliance with the previous criteria ${ }^{[12-13]}$, we regulated $\mathrm{DEGs}$ as differentially expressed with $\log \mathrm{FC}>1$ (upregulated genes) or $\log \mathrm{FC}<-1$ (downregulated genes). In the meantime, the adjusted $P$ value $<0.05$ was regarded statistically significant to reduce the false positive rate. Moreover, after downloading the correlative data of TXT files, we employed visual hierarchical cluster analysis to show the heatmap and volcano plot of AR and no rejection samples via ImageGP (http://www.ehbio.com /ImageGP/index.php/Home/Imdex/index.html)

\subsection{Gene ontology (GO) and Kyoto Encyclopedia of Genes and Genomes (KEGG) Pathway Analysis of} DEGs $\mathrm{GO}$ analysis is a public framework which can note on genes and gene products involving biological pathways (BP), molecular function (MF) and cellular components (CC ${ }^{[14]}$. The KEGG pathway is a set of databases manifesting genomes and biological pathways related to diseases and drugs. KEGG is essentially a resource to get an systematic understanding of biological functions and even certain highlevel genome information ${ }^{[15]}$. In our study, the GO and KEGG analysis was accomplished by the database for Annotation, Visualization and Integrated Discovery (DAVID, http://david.ncifcrf.gov) (version 6.7), which has contained a great many biological data and concerned analysis methods, and then supplied tools about the biological function annotation information for lots of genes or proteins ${ }^{[16]}$. $P<0.05$ was regarded as the cutoff criterion with significant difference. Besides, we displayed the scatter plot of the $\mathrm{BP}, \mathrm{MF}, \mathrm{CC}$ and KEGG pathway of these DEGs using ImageGP.

\subsection{Protein-Protein Interaction(PPI) Network and Module Analysis We used the PPI network to illustrate} the core hub genes and main gene modules between transplant patients with and without AR. Search Tool for the Retreval of Interacting Genes(STRING) is an well-known database to assess and integrate the PPI information(http://www.stringdb.org/), which includes physical and functional associations. It covered 24584628 proteins from 5090 organisms in STRING version $11.0^{[17]}$. We applied DEGS to identify the interactional associations among them using STRING. Furthermore, we built a PPI network through Cytoscape software platform. Then, we regarded the maximum number of interactors $=0$, confidence score $\geq 0.4$ as the curoff criterion. Moreover, the Molecular Complex Detection (MCODE) app was perfomed to reveal modules of the PPI network in Cytoscape in accordance with degree cutoff $=2, \mathrm{k}$-core $=2$, node score cutoff $=0.2$, and max. depth $=100$. Top 12 hub genes were selected in line with the ranking order of connectivity degree via Cytoscape with maximum number of interactors $\leq 5$ and 
confidence score $\geq 0.4$. Besides, GO and KEGG pathway analyses were applied to demonstrate the potential information.

2.5 Gene Set Enrichment Analysis (GSEA) 6 AR samples from GSE1563 were divided into two groups (high vs. low) on the basis of expression level of several hub genes, and median expression value was considered as the cutoff point. In order to reveal the potential function of these related hub genes, GSEA(http://software.broadinstitute.org/gsea/index.jsp) was performed between the two groups. Gene sets database c2.cp.kegg.v7.1.symbols.gmt, c5.bp.v7.1. symbols.gmt, c5.cc.v7.1. symbols.gmt, c5.mf.v7.1. symbols.gmt were selected as the reference gene sets. FDR $<0.05$,|enrichment score(ES)|>0.5 and gene size $\geq 100$ were regarded as the cutoff criteria.

2.6 Statistical Analysis All obtained data were reported as means $\pm S D$ (standard deviation) Statistical significance was assessed by the two tailed Student's t-test via SPSS 19.0 software. A difference of $P<$ 0,05 was considered statistically significant.

\section{Results}

3.1 Identification of DEGs In our study, a total of 6 kidney transplant patients undergoing AR and 9 patients with well-functioning transplant with no clinical evidence of rejection were analyzed. We used the GEO2R online analysis tool on the basis of default parameters to screen the DEGs with logFC $\leq-1$ or $\operatorname{logFC} \geq 1$ and adjusted $P$ value $<0.05$ and as the cut-off criteria. Through analysis of GSE1563, a total of 347 DEGs were captured, 301 of which were upregulated genes while 46 were downregulated genes. The expression ratio of these DEGs were shown in the volcano plot (Fig. 2A). Meanwhile, the top30 upregulated genes and top30 downregulated genes between transplant patient with and without AR were displayed in the heatmap (Fig. 2B). In these 347 DEGs, the top5 up-regulated genes including CD9, SLC39A6, ATP5F1, MORF4L2 and RYK as the top5 down-regulated genes were MAPK8, HLCS, CCL7, CCL5 and S100A9. The gene titles and biological functions of these 10 genes were displayed in Table 1. 


\begin{tabular}{|c|c|c|c|c|}
\hline DEGs & Gene title & $\begin{array}{l}\text { Gene } \\
\text { symbol }\end{array}$ & LogFC & Biological function \\
\hline \multirow[t]{5}{*}{$\begin{array}{l}\text { Up- } \\
\text { regulated }\end{array}$} & CD9 molecule & CD9 & 1.90 & $\begin{array}{l}\text { Cell differentiation, adhesion, } \\
\text { and signal transduction }\end{array}$ \\
\hline & $\begin{array}{l}\text { solute carrier family } 39 \text { member } \\
6\end{array}$ & SLC39A6 & 1.88 & $\begin{array}{l}\text { Zinc transport and } \\
\text { lymphocyte activation }\end{array}$ \\
\hline & $\begin{array}{l}\text { ATP synthase, H+ transporting, } \\
\text { mitochondrial F0 complex, } \\
\text { subunit B1 }\end{array}$ & ATP5F1 & 1.86 & Participate in ATP synthesis \\
\hline & mortality factor 4 like 2 & MORF4L2 & 1.84 & $\begin{array}{l}\text { abnormal nuclear } \\
\text { morphology and cell death. }\end{array}$ \\
\hline & receptor like tyrosine kinase & RYK & 1.77 & $\begin{array}{l}\text { stimulating Wnt signaling } \\
\text { pathways }\end{array}$ \\
\hline \multirow[t]{5}{*}{$\begin{array}{l}\text { Down- } \\
\text { regulated }\end{array}$} & S100 calcium binding protein A9 & S100A9 & -1.51 & $\begin{array}{l}\text { Regulating cell cycle } \\
\text { progression and } \\
\text { differentiation }\end{array}$ \\
\hline & C-C motif chemokine ligand 5 & CCL5 & -1.50 & $\begin{array}{l}\text { release of histamine from } \\
\text { basophils and activating } \\
\text { eosinophils }\end{array}$ \\
\hline & $\mathrm{C}-\mathrm{C}$ motif chemokine ligand 7 & CCL7 & -1.48 & $\begin{array}{l}\text { Involving in inflammation } \\
\text { and metastasis }\end{array}$ \\
\hline & holocarboxylase synthetase & HLCS & -1.42 & $\begin{array}{l}\text { gluconeogenesis, fatty acid } \\
\text { synthesis and branched } \\
\text { chain amino acid catabolism }\end{array}$ \\
\hline & $\begin{array}{l}\text { mitogen-activated protein kinase } \\
8\end{array}$ & MAPK8 & -1.33 & $\begin{array}{l}\text { T cell proliferation, apoptosis } \\
\text { and differentiation }\end{array}$ \\
\hline
\end{tabular}

Table 1

Top5 up-regulated and down-regulated differentially expressed gene in patients with AR

3.2 GO Enrichment Analysis To obtain a more particular and in-depth knowledge of these captured DEGs, we applied DAVID to analyze significantly enriched GO function of these upregulated DEGs and downregulated DEGs. The results displayed that the up-regulated DEGs in BP were mainly enriched in protein ubiquitination, protein stabilization, response to drug, chemokine-mediated signaling pathway and inflammatory response to antigenic stimulus whereas the downregulated DEGs in BP were enriched in innate immune response, immune response, neutrophil chemotaxis, cellular response to interleukin-1 and positive regulation of inflammatory response. Concerning MF, the up-regulated DEGs were primarily enriched in poly (A) RNA binding, ATP binding, nucleotide binding, protein kinase activity and transcription corepressor activity while the down-regulated DEGs were enriched in protein binding, Toll-like receptor 4 binding, arachidonic acid binding, $\mathrm{C}-\mathrm{C}$ motif chemokine receptor 1 (CCR1) chemokine receptor binding and the receptor of advanced glycation end-products (RAGE) receptor binding. Besides, CC analysis 
indicated that the up-regulated DEGs were principally enriched in cytoplasm, nucleus, extracellular exosome, nucleoplasm and nucleolus. The down-regulated DEGs were related to extracellular space, cytosol, extracellular region, extracellular exosome and cytoskeleton (Table 2\&Figure 3A, B and C). 


\begin{tabular}{|c|c|c|c|c|c|}
\hline Expression & Category & Term & Count & $\%$ & PValue \\
\hline \multirow[t]{15}{*}{$\begin{array}{l}\text { Up- } \\
\text { regulated }\end{array}$} & GOTERM_BP_DIRECT & $\begin{array}{l}\text { GO:0042787 protein } \\
\text { ubiquitination involved in } \\
\text { ubiquitin-dependent protein } \\
\text { catabolic process }\end{array}$ & 7 & 0.02 & 0.01 \\
\hline & GOTERM_BP_DIRECT & GO:0050821 protein stabilization & 6 & 0.01 & 0.03 \\
\hline & GOTERM_BP_DIRECT & G0:0042493 response to drug & 5 & 0.01 & 0.03 \\
\hline & GOTERM_BP_DIRECT & $\begin{array}{l}\text { GO:0070098 chemokine- } \\
\text { mediated signaling pathway }\end{array}$ & 4 & 0.01 & 0.04 \\
\hline & GOTERM_BP_DIRECT & $\begin{array}{l}\text { GO:0002437 inflammatory } \\
\text { response to antigenic stimulus }\end{array}$ & 3 & 0.01 & 0.01 \\
\hline & GOTERM_CC_DIRECT & GO:0005737 cytoplasm & 62 & 0.13 & $<0.01$ \\
\hline & GOTERM_CC_DIRECT & GO:0005634 nucleus & 56 & 0.12 & 0.03 \\
\hline & GOTERM_CC_DIRECT & $\begin{array}{l}\text { G0:0070062 extracellular } \\
\text { exosome }\end{array}$ & 52 & 0.11 & 0.01 \\
\hline & GOTERM_CC_DIRECT & GO:0005654 nucleoplasm & 35 & 0.08 & $<0.01$ \\
\hline & GOTERM_CC_DIRECT & Go:0005730 nucleolus & 22 & 0.05 & $<0.01$ \\
\hline & GOTERM_MF_DIRECT & $\begin{array}{l}\text { GO:0044822 poly(A) RNA } \\
\text { binding }\end{array}$ & 39 & 0.08 & $<0.01$ \\
\hline & GOTERM_MF_DIRECT & G0:0005524 ATP binding & 30 & 0.07 & 0.04 \\
\hline & GOTERM_MF_DIRECT & GO:0000166 nucleotide binding & 11 & 0.02 & 0.03 \\
\hline & GOTERM_MF_DIRECT & $\begin{array}{l}\text { GO:0004672 protein kinase } \\
\text { activity }\end{array}$ & 8 & 0.02 & 0.02 \\
\hline & GOTERM_MF_DIRECT & $\begin{array}{l}\text { GO:0003714 transcription } \\
\text { corepressor activity }\end{array}$ & 6 & 0.01 & 0.04 \\
\hline \multirow[t]{7}{*}{$\begin{array}{l}\text { Down- } \\
\text { regulated }\end{array}$} & GOTERM_BP_DIRECT & $\begin{array}{l}\text { G0:0045087 innate immune } \\
\text { response }\end{array}$ & 6 & 0.29 & $<0.01$ \\
\hline & GOTERM_BP_DIRECT & GO:0006955 immune response & 5 & 0.24 & $<0.01$ \\
\hline & GOTERM_BP_DIRECT & $\begin{array}{l}\text { GO:0030593 neutrophil } \\
\text { chemotaxis }\end{array}$ & 4 & 0.20 & $<0.01$ \\
\hline & GOTERM_BP_DIRECT & $\begin{array}{l}\text { Go:0071347 cellular response to } \\
\text { interleukin-1 }\end{array}$ & 4 & 0.20 & $<0.01$ \\
\hline & GOTERM_BP_DIRECT & $\begin{array}{l}\text { GO:0050729 positive regulation } \\
\text { of inflammatory response }\end{array}$ & 4 & 0.20 & $<0.01$ \\
\hline & GOTERM_CC_DIRECT & Go:0005615 extracellular space & 10 & 0.49 & $<0.01$ \\
\hline & GOTERM_CC_DIRECT & GO:0005829 cytosol & 10 & 0.49 & $<0.01$ \\
\hline
\end{tabular}




\begin{tabular}{lllll|} 
GOTERM_CC_DIRECT & G0:0005576 extracellular region & 8 & 0.39 & $<0.01$ \\
\hline GOTERM_CC_DIRECT & $\begin{array}{l}\text { G0:0070062 extracellular } \\
\text { exosome }\end{array}$ & 8 & 0.39 & 0.02 \\
\hline GOTERM_CC_DIRECT & G0:0005856 cytoskeleton & 3 & 0.15 & 0.05 \\
\hline GOTERM_MF_DIRECT & G0:0005515 protein binding & 14 & 0.69 & 0.04 \\
\hline GOTERM_MF_DIRECT & $\begin{array}{l}\text { G0:0035662 Toll-like receptor } 4 \\
\text { binding }\end{array}$ & 2 & 0.10 & $<0.01$ \\
\hline GOTERM_MF_DIRECT & $\begin{array}{l}\text { GO:0050544 arachidonic acid } \\
\text { binding }\end{array}$ & 2 & 0.10 & 0.01 \\
\hline GOTERM_MF_DIRECT & $\begin{array}{l}\text { GO:0031726 CCR1 chemokine } \\
\text { receptor binding }\end{array}$ & 2 & 0.10 & 0.01 \\
\hline GOTERM_MF_DIRECT & $\begin{array}{l}\text { GO:0050786 RAGE receptor } \\
\text { binding }\end{array}$ & 2 & 0.10 & 0.01 \\
\hline
\end{tabular}

Table 2

Gene ontology analysis of differentially expressed genes in transplant patients with AR 


\begin{tabular}{|c|c|c|c|c|c|}
\hline Category & Term & Count & $\%$ & PValue & Genes \\
\hline \multirow[t]{5}{*}{$\begin{array}{l}\text { Up- } \\
\text { regulated }\end{array}$} & ptr03013: RNA transport & 10 & 0.02 & $<0.01$ & $\begin{array}{l}\text { SUMO3, NCBP2, XP01, } \\
\text { NUP153, SUMO1, NUP50, } \\
\text { SRRM1, RNPS1, CASC3, PNN }\end{array}$ \\
\hline & $\begin{array}{l}\text { ptr05203: Viral } \\
\text { carcinogenesis }\end{array}$ & 9 & 0.02 & 0.05 & $\begin{array}{l}\text { KRAS, YWHAH, DDX3X, IL6ST, } \\
\text { UBE3A, RB1, PMAIP1, RBPJ, } \\
\text { DLG1 }\end{array}$ \\
\hline & $\begin{array}{l}\text { ptr01130: Biosynthesis of } \\
\text { antibiotics }\end{array}$ & 9 & 0.02 & 0.05 & $\begin{array}{l}\text { ACADM, DLD, TGDS, PFKP, } \\
\text { ACLY, ACAT1, OAT, PCCA, PCK1 }\end{array}$ \\
\hline & $\begin{array}{l}\text { ptr04068: FoxO signaling } \\
\text { pathway }\end{array}$ & 7 & 0.02 & 0.04 & $\begin{array}{l}\text { GABARAPL1, KRAS, TGFBR2, } \\
\text { BNIP3, CCNG2, CHUK, PCK1 }\end{array}$ \\
\hline & $\begin{array}{l}\text { ptr03015: mRNA } \\
\text { surveillance pathway }\end{array}$ & 6 & 0.01 & 0.04 & $\begin{array}{l}\text { NCBP2, PPP2CB, SRRM1, } \\
\text { RNPS1, CASC3, PNN }\end{array}$ \\
\hline \multirow[t]{5}{*}{$\begin{array}{l}\text { Down- } \\
\text { regulated }\end{array}$} & $\begin{array}{l}\text { hsa05168: Herpes } \\
\text { simplex infection }\end{array}$ & 4 & 0.20 & $<0.01$ & MAPK8, CCL5, TAF6L, HLA-F \\
\hline & hsa05164: Influenza A & 3 & 0.15 & 0.02 & ACTB, MAPK8, CCL5 \\
\hline & $\begin{array}{l}\text { hsa04621: NOD-like } \\
\text { receptor signaling } \\
\text { pathway }\end{array}$ & 2 & 0.10 & 0.04 & MAPK8, CCL5 \\
\hline & $\begin{array}{l}\text { hsa05416: Viral } \\
\text { myocarditis }\end{array}$ & 2 & 0.10 & 0.02 & ACTB, HLA-F \\
\hline & hsa05131: Shigellosis & 2 & 0.10 & 0.03 & ACTB, MAPK8 \\
\hline
\end{tabular}

Table 3

KEGG pathway analysis of differentially expressed genes in transplant patients with AR

3.3 KEGG pathway analysis In order to acquire a more detailed information about the essential pathway of those selected DEGs, we also analyzed the most significantly enriched KEGG pathway of the upregulated and down-regulated DEGs via DAVID, which is uncovered in Table 4 and Fig. 3D. The upregulated DEGs were involved in RNA transport, Viral carcinogenesis, Biosynthesis of antibiotics, The forkhead box $\mathrm{O}$ (FoxO) signaling pathway and mRNA surveillance pathway. By contrast, the downregulated DEGs, namely ACTB, MAPK8, CCL5 and HLA-F were mainly enriched in Herpes simplex infection, Influenza A, NOD-like receptor signaling pathway, Viral myocarditis and Shigellosis. 


\begin{tabular}{|llll|}
\hline Gene & Degree of connectivity & P value & LogFC \\
\hline MAPK8 & 26 & $1.25 \mathrm{E}-02$ & -1.33 \\
\hline APP & 26 & $1.28 \mathrm{E}-02$ & 1.32 \\
\hline ACTB & 26 & $1.68 \mathrm{E}-02$ & -1.04 \\
\hline HNRNPU & 22 & $2.58 \mathrm{E}-04$ & 1.07 \\
\hline CYCS & 22 & $1.77 \mathrm{E}-03$ & 1.16 \\
\hline XP01 & 21 & $2.41 \mathrm{E}-03$ & 1.06 \\
\hline SRRM1 & 21 & $5.11 \mathrm{E}-04$ & 1.18 \\
\hline SUM01 & 21 & $7.94 \mathrm{E}-05$ & 1.13 \\
\hline KRAS & 21 & $1.34 \mathrm{E}-04$ & 1.05 \\
\hline RNPS1 & 20 & $6.62 \mathrm{E}-05$ & 1.01 \\
\hline SNRPD3 & 18 & $3.27 \mathrm{E}-05$ & 1.29 \\
\hline PARP1 & 18 & $1.71 \mathrm{E}-04$ & 1.23 \\
\hline NCBP2 & 18 & $6.70 \mathrm{E}-04$ & 1.12 \\
\hline LUC7L3 & 17 & $3.33 \mathrm{E}-04$ & 1.06 \\
\hline RBM39 & 17 & $1.68 \mathrm{E}-05$ & 1.42 \\
\hline SFPQ & 17 & $4.78 \mathrm{E}-03$ & 1.10 \\
\hline CCL5 & 17 & $2.28 \mathrm{E}-03$ & -1.50 \\
\hline HMGB1 & 17 & $1.43 \mathrm{E}-05$ & 1.46 \\
\hline
\end{tabular}

Table 4

Top 18 hub genes with higher degree of connectivity

3.4 Hub genes and Module Screening from PPI Network In order to identify the core genes in those DEGs, we apply the STRING online tool to detect 261 nodes with 845 PPI relationships, which accounted for about $95.7 \%$ of these selected DEGs (Fig. 4A). On the basis of the degree of connectivity, we constructed the PPI network and chose the top 18 hub genes (Table 4). The top 18 hub genes with a higher degree of connectivity in AR are as follows: MAPK8, APP, ACTB, HNRNPU, CYCS, XPO1, SRRM1, SUM01, KRAS, RNPS1, SNRPD3, PARP1, NCBP2, LUC7L3, RBM39, SFPQ, CCL5 and HMGB1. Among these 18 hub genes, MAPK8, ACTB and CCL5 were significantly down-regulated while APP, HNRNPU, CYCS, XPO1, SRRM1, SUM01, KRAS, RNPS1, SNRPD3, PARP1, NCBP2, LUC7L3, RBM39, SFPQ and HMGB1 were up-regulated. The 18 hub genes could interact with 142 genes directly. Besides, MAPK8, APP and ACTB acted as the most intensive gene, all of which could interact with 23 up-regulated genes and 3 down-regulated genes 
respectively. Interestingly, among these hub genes also displayed strong interactions (Fig. 4B). For instance, HMGB1 could directly interact with various genes (ACTB, APP, MAPK8, PARP1, XPO1 and CYCS), and meanwhile XPO1 could interact with 5 genes (HMGB1, SUM01, SNRPD3, NCBP2 and HNRNPU). Applying the GO function and KEGG pathway analysis of these hub genes, we uncovered MAPK8, XPO1, CYCS and NCBP2 are the 4 high-degree-of-connectivity genes related with RNA transport and immune response. Taken together, these results indicated that these hub genes, especially MAPK8, XPO1, CYCS and HMGB1 may have an essential effect in AR, which are linked with each other tightly (Table 5).

\begin{tabular}{|llllll|}
\hline Term & Count & $\%$ & PValue & Genes & FDR \\
\hline cfa05164: Influenza A & 5 & 0.16 & $<0.01$ & $\begin{array}{l}\text { ACTB, XP01, CYCS, MAPK8, } \\
\text { CCL5 }\end{array}$ & 0.26 \\
\hline cfa03013: RNA transport & 5 & 0.16 & $<0.01$ & $\begin{array}{l}\text { NCBP2, XP01, SUM01, } \\
\text { SRRM1, RNPS1 }\end{array}$ & 0.28 \\
\hline $\begin{array}{l}\text { cfa05210: Colorectal cancer } \\
\text { ffa03015: mRNA surveillance }\end{array}$ & 3 & 0.10 & 0.01 & KRAS, CYCS, MAPK8 & 7.40 \\
$\begin{array}{l}\text { pathway } \\
\text { cfa03040: Spliceosome }\end{array}$ & 3 & 0.10 & 0.01 & NCBP2, SRRM1, RNPS1 & 15.06 \\
\hline
\end{tabular}

Table 5

KEGG pathway analysis of top 18 hub genes with higher degree of connectivity

Moreover, we applied MCODE plug-in to reveal the highest modules in the PPI network. We selected the top 3 modules, the scores (Densityx\#Nodes) of which were $\geq 4$. Then, we analyzed the Go function and KEGG pathway enrichment to uncover that Module 1 and Module 3 was associated with RNA transport and mRNA surveillance pathway, while Module 2 was related to inflammatory response and several signaling pathway (Fig. 5 and Table 6). 


\begin{tabular}{|c|c|c|c|c|}
\hline Module & Term & PValue & FDR & Genes \\
\hline \multirow[t]{5}{*}{$\begin{array}{l}\text { Module } \\
1\end{array}$} & $\begin{array}{l}\text { protein ubiquitination involved in ubiquitin- } \\
\text { dependent protein catabolic process }\end{array}$ & $<0.01$ & 0.11 & $\begin{array}{l}\text { CUL2, CUL4A, DZIP3, } \\
\text { UBE3A, TRIP12 }\end{array}$ \\
\hline & RNA transport & $<0.01$ & $<0.01$ & $\begin{array}{l}\text { NCBP2, SUMO3, XP01, } \\
\text { SUMO1, NUP153, } \\
\text { NUP50, CASC3, PNN }\end{array}$ \\
\hline & Ubiquitin mediated proteolysis & $<0.01$ & 1.01 & $\begin{array}{l}\text { CUL2, CUL4A, UBE3A, } \\
\text { UBA3, TRIP12 }\end{array}$ \\
\hline & Nucleotide excision repair & $<0.01$ & 0.39 & $\begin{array}{l}\text { RPA1, ERCC5, CUL4A, } \\
\text { GTF2H2 }\end{array}$ \\
\hline & mRNA surveillance pathway & $<0.01$ & 3.06 & $\begin{array}{l}\text { NCBP2, PPP2CB, CASC3, } \\
\text { PNN }\end{array}$ \\
\hline \multirow[t]{5}{*}{$\begin{array}{l}\text { Module } \\
2\end{array}$} & inflammatory response & $<0.01$ & 2.60 & $\begin{array}{l}\text { HMGB1, PPBP, CCL5, } \\
\text { CCL4 }\end{array}$ \\
\hline & chemokine-mediated signaling pathway & $<0.01$ & 1.65 & PPBP, CCL5, CCL4 \\
\hline & neutrophil chemotaxis & $<0.01$ & 2.76 & PPBP, CCL5, CCL4 \\
\hline & Toll-like receptor signaling pathway & $<0.01$ & 1.46 & $\begin{array}{l}\text { MAPK8, CCL5, CCL4, } \\
\text { SPP1 }\end{array}$ \\
\hline & NOD-like receptor signaling pathway & 0.01 & 6.09 & MAPK8, BIRC3, CCL5 \\
\hline \multirow{2}{*}{$\begin{array}{l}\text { Module } \\
3\end{array}$} & mRNA surveillance pathway & 0.01 & 2.98 & SRRM1, RNPS1 \\
\hline & RNA transport & 0.02 & 5.27 & SRRM1, RNPS1 \\
\hline
\end{tabular}

\section{Table 6}

The enriched pathway of top 3 modules

3.5 Gene Set Enrichment Analysis Furthermore, GSEA was performed to map into GO analysis and KEGG pathway database in order to acquire further insight into the function of the hub gene. According to the cut-off criteria FDR $<0.05$,|enrichment score(ES)|>0.5 and gene size $\geq 100,6$ functional gene sets were enriched totally, which particularly focused on pathways linked with immune response, RNA transport as well as oxidoreductase activity of donors. Six pathway were respectively"myeloid leukocyte mediated immunity", "regulation of humoral immune response", "oxidoreductase activity acting on the aldehyde or oxo group of donors", "mature B cell differentiation involved in immune response", "regulation of B cell proliferation", "ncRNA export from nucleus"(Fig. 6).

\section{Discussion}

In recent years, although the overall incidence and prevalence of acute allograft in kidney transplantation have decreased, it is also a vital reason which leads to graft dysfunction and even death ${ }^{[18]}$. Presently, as 
the result of the usage of more effective and advanced immunosuppressive drugs, the incidence of acute rejection in the first year is reduced to approximately $7.9 \%{ }^{[19]}$. Currently, renal biopsy remains the gold standard for the specific injury of AR. However, in consideration of its side effects, performing serial renal biopsies are impractical at the same periodicity as blood or urine examination. Besides, Dharnidharka and his colleagues found that cellular and molecular events of AR occur before the rise of the clinical biomarker like serum creatinine as well as according to serum creatinine elevated, clinical doctors can't distinguished a superimposed AR from chronic allograft injury progression, which suggests that functional markers like serum creatinine for glomerular filtration are not as specific as injury markers ${ }^{[20]}$. Therefore, it is be in urgent need of more accurate and less invasive methods to diagnose AR so that potent immunosuppressive can be applied in time. Although peripheral blood biomarkers are not as general as that in cardiopathy and tumors due to the complicated molecular mechanisms, it doesn't mean that it makes no sense to perform peripheral blood biomarker to diagnose. Roedder et al. ${ }^{[21]}$ used a kidney transplant cohort called the Assessment of Acute Rejection in Renal Transplantation (AART)study to verify a 17-gene signature in the peripheral blood that uncovered an area under curve (AUC) of 0.92 to predict AR. The above studies revealed the potential of peripheral blood biomarkers in diagnosis of AR. In our study, we implemented a synthetic investigation on the expression profiling of PBL from transplant patients with AR which included 6 kidney transplant patients undergoing AR and 9 patients with wellfunctioning transplant with no clinical evidence of rejection from the GEO database of GSE1563. By the way of defining the absolute value of the logarithm (base 2) fold change (log FC) greater than 2, We identified totally 347 DEGs (account for $2.75 \%$ of all genes) where 301 genes were upregulated and 46 were downregulated. In order to have a further detecting and analyzing these DEGs, we performed GO function, KEGG pathway to select 10 sensitive genes and top 18 hub genes were detected via PPI network and connectivity analysis of these DEGs. Additionally, we also applied GSEA to reveal the potential mechanisms of AR through these hub genes.

There were a great many factors related to transplant patients occurring $A R$, but the primary mechanism to contribute to the AR is still controversial, which is the reason that why it is difficult to diagnose and treat AR. According to our study, we used GO term enrichment and PPI analysis to uncover that the DEGs were closely interrelated with immune response and inflammatory process, especially downregulated DEGs. It is clear that the biological process associated with immunity plays an important role in the development of AR.

On the basis of pathophysiology, AR fall into two categories, which are antibody-mediated rejection (ABMR) and acute T-cell mediated rejection (TCMR) respectively ${ }^{[22]}$. On the one hand, Inflammation of glomeruli and peritubular capillary are always attributed to ABMR. Patients with ABMR usually verifies evidence of circulating donor-specific alloantibodies and immunological evidence of antibody-mediated injury ${ }^{[23]}$. It is reported that acute ABMR occur in about $7 \%$ of patients and it can be up to $50 \%$ in patients with human leukocyte antigen (HLA)-incompatible transplants ${ }^{[24-25]}$. It is much worse plenty of these patients may develop transplant glomerulopathy or chronic ABMR and have beyond a quadruple risk of graft loss compared to control groups ${ }^{[26]}$. On the other hand, TCMR is characterized by lymphocytic 
infiltration of the renal tubules, interstitium and arterial intima ${ }^{[27]}$. Zoghby et al. proved that TCMR is one of the most important and serious complications occurring in the renal transplant patients and it will cause graft loss and permanent impairment of graft function if not early diagnosis ${ }^{[28]}$. Therefore, we found that both ABMR and TCMR are equally essential for renal transplant patients which need to early detection and treatment.

In our GO analysis, it is indicated that DEGs, especially down-regulated DEGs, were mainly associated with immune response as well as neutrophil chemotaxis, innate immune response and inflammatory response. To our knowledge, ABMR is linked with antibodies against foreign donor antigens, especially HLA antigen which contributes to impairing the allograft by the way of activating the complementdependent pathway and independent mechanisms recruiting NK cells, macrophages, polymorphonuclear cells and platelets to attack the allograft ${ }^{[29]}$. According to this mechanism, we uncovered that there were 3 genes (MAPK8, CCL5 and HMGB1) possibly related to the immune response occurring in ABMR. Haylett et al. discovered that MHC class II molecules can adjust mitogen-activated protein kinase 8 (MAPK8) pathway and the expression of c-Fos in B cells which can induce B cell to release the antibody ${ }^{[30]}$. In another study, MAPK8 was related to the release of BECN1 form its complex with BCL2 apoptosis regulator (BCL2), which in turn results in macrophages, an important class of antigen-presenting cells that activated adaptive immune responses autophagy ${ }^{[31]}$. Besides, it is reported that Fingolimod can increase the $\mathrm{C}-\mathrm{C}$ motif chemokine ligand 5 (CCL5) in peripheral blood to inhibit the egress of lymphocyte involved in antigen presentation, which restrain B cells for humoral immunity ${ }^{[32]}$. As the same result, Sullivan et al. demonstrated endogenous CCL5 had a neutralization effect and inhibited B cell proliferation and IgM secretion when B cells stimulation ${ }^{[33]}$. As for high mobility group box 1 (HMGB1), through Sprague-Dawley rat hearts transplanted heterotopically into BALB/c mice, it is indicated HMGB1 regulated B cell activation and IFN- $\gamma$ and IL-17A production which plays an important role in mediating acute xenograft rejection ${ }^{[34]}$. Thus, our results suggested the activation and proliferation of $B$ cell and other antigen-presenting cells (APC) attach great importance to the development of ABMR via MAPK8, CCL5 and HMGB1 and further affect the severity of AR.

In TCMR, APC recognize the foreign donor antigens in the transplanted organ via various pathways to activate the recipient's lymphocytes. As a result, T cells were activated and infiltrated which can damage the allograft. Thus, the most important part in TCMR is the activation of T cell. It is reported that some molecules can shared the important rejection mechanisms between B cells and NK cells in ABMR and effector T cells in TCMR ${ }^{[35]}$. Therefore. We predicted the mechanisms of T lymphocytes activity and recruitment may also be associated with those 3 hub genes (MAPK8, CCL5 and HMGB1) through GO and KEGG analysis. MAPK8 signaling has been implicated in multiple T cell functions. Similar to MAPK8 activation response in B cells, the MAPK8 is also synergistically activated by co-stimulation of the TCR with antibodies to its $\mathrm{CD} 3$ component and the $\mathrm{CD} 28$ auxiliary receptor ${ }^{[36]}$. Meanwhile, the activation of MAPK8 in T cells can inhibit the proliferation and infiltration due to neutralization. Additionally, the misbalanced level of chemokines like CCL5 is associated with the activation and function of cytotoxic T lymphocytes and may constrain CD $8+T$ cell out of lymphoid organs ${ }^{[32,37]}$. Furthermore, it is reported 
that HMGB1 can recruits cells across endothelial barriers and induced the production of tumor-necrosis (TNF) and interferon, which is an nuclear weapon in the immune arsenal ${ }^{[38]}$. In short, from our perspective based on the $\mathrm{GO}$ and KEGG analyses, the regulation of immune-related genes involving MAPK8, CCL5 and HMGB1 can play an essential role in ABMR and TCMR which equally have an important effect in the occurrence and progression of AR. Thus, the detection of these target genes in peripheral blood may be a potential candidate for early diagnose of AR.

The PPI network could construct an obvious framework to have a better comprehension of the function of the proteome ${ }^{[39]}$. Through the enriched pathway of top 3 modules, we indicated that the interactions among the proteins in AR are specifically associated with inflammatory response, chemokine-mediated signaling pathway and neutrophil chemotaxis, which is as the same results as above. It emphasizes again that the interaction of immune-related genes can regulate the humoral immunity and cellular immunity associated with AR. Surprisingly, from enriched pathway of Modules 1, we found that some related proteins (NCBP2 and XPO1) in AR are also linked with RNA transport and mRNA surveillance pathway. It implies that RNA transport will be another mechanism of AR development. Coincidentally, we performed GSEA and discovered that non-coding RNA (ncRNA) export from nucleus is one of the key biological processes of AR. We applied DAVID to uncover the function of 18 hub genes and verified the same evidence that nuclear cap binding protein subunit 2 (NCBP2) and exportin 1 (XPO1) have a relationship with RNA transport. Therefore, we speculated that RNA transport can regulate some ncRNA transport which can be detected for diagnosis. Anglicheau et al. demonstrated that the levels of six miRNAs (miR-142-5p, miR-155, miR-223, miR-10b, miR-30a-3p, and let-7c) can become the biomarkers to diagnose AR, among which miR-142-5p, miR-155 and miR-233 can predicted AR with above $90 \%$ specificity and sensitivity ${ }^{[40]}$. Büssing et al. used C. elegans and Drosophila to prove that as a nuclear export receptor, XPO1 interacted with NCBP2 which is a subunit of the nuclear cap-binding complex to promote the pri-miRNA to pre-miRNA processing and induce nuclear export of miRNAs ${ }^{[41]}$. The above studies further consolidated our analysis, which revealed that NCBP2 and XPO1 were inextricably linked with the process of ncRNA, especially miRNA transport. Hence, monitoring the ncRNA transport genes and even specific miRNA of AR is of great importance for the diagnosis of AR.

Moreover, when we sifted the GSEA carefully, we uncovered that the development of AR may also be attributed to oxidoreductase activity acting on the aldehyde or oxo group of donors associated with amyloid beta precursor protein (APP). A part of oxidoreductase like Xanthine oxidase can increase the oxidative radical production, which may result in the dysregulation of redox homeostasis and excessive generation of reactive oxygen species (ROS), culminating in oxidative stress and the linked oxidative damage of cellular components ${ }^{[42]}$. Oxidative stress and ROS play an essential role in the pathogenesis of AR. Additionally, oxidative stress can activate autophagy, which can bring about the promotion of inflammatory response indirectly and enhance the progression of AR. APP is an oxidative stress responsive molecule. With APP overexpression, it will damage the structure of mitochondria and increase ROS production, which enhanced oxidative stress ${ }^{[43]}$. Besides, Sennvik et al. used western blotting to compare the cerebrospinal fluid from Alzheimer's disease patients with healthy individual and revealed 
APP underwent posttranslational proteolytic processing by $a-, \beta-, \gamma$-secretases, which can produce soluble

amyloid protein or APP components with amyloidogenic characters to induce oxidative stress ${ }^{[44]}$. It is consistent with our GO and KEGG analyses that protein stabilization is an important process of AR. Therefore, we hypothesized that APP is linked with oxidative stress resulting in protein hydrolysis and inflammatory response. Detecting this gene can early discover the subtle injury of AR in order to deal with it as early as possible.

Conclusively, applying a series of bioinformatics analysis, we provide a comprehensive and novel demonstration of gene expression profiles to recognize DEG expressing in peripheral blood, which may play critical roles in the occurrence and development in renal transplant patients with AR. Genes implicated with immune, inflammation, RNA transport as well as oxidative stress were apparently altered in AR patients, among which MAPK8, CCL5 and HMGB1 play core roles in the immune response of AR including ABMR and TCMR. Additionally, the interaction of NCBP2 and XPO1 will become the new mechanism to speculate the ncRNA transport of AR and may discover more novel miRNA of AR via the mechanism of these two genes. Furthermore, the graft dysfunction and fibrosis were associated with oxidative stress linked with APP in AR, which can be a promising strategy to diagnose and treat AR from another perspective. Taken together, we found that MAPK8, CCL5, HMGB1, NCBP2, XPO1 and APP may be regarded as potential serum biomarkers and targets of therapy. Certainly, we need further experiments and investigations to make sure the correlation of these dysregulated genes in order to allow these biomarkers to be applied more ordinarily in clinic. We expect this kind of analyzing method will provide more valuable and precise information for our future study on the molecular mechanisms of AR and offer more accurate evidences for detection of new diagnosis biomarkers and therapeutic strategies.

\section{Declarations}

\section{Ethics approval and consent to participate}

Not applicable

\section{Consent for publication}

Not applicable

\section{Availability of data and materials}

All data generated or analysed during this study are included in this published article

\section{Competing interests}

The authors declare that they have no competing interests 


\section{Funding}

This study was supported by the National Natural Science Foundation of China (nos. 81673503 and 30973582), and the Medical Science Advancement Program (Clinical Medicine) of Wuhan University (no. TFLC 2018003)

\section{Authors' contributions}

$\mathrm{HZ}$ supported the main conception and design work of this manuscript and was a main contributor in writing the manuscript. LL supported a part of design and was a contributor in writing the manuscript. QY contributed some design work and revised the manuscript. All authors read and approved the final manuscript and have agreed both to be personally accountable for their own contributions and ensured that questions related to the accuracy or integrity of any part of the work.

\section{Acknowledgements}

The authors thank the professors and students from Zhongnan Hospital of Wuhan University and the Institute of Hepatobiliary Diseases of Wuhan University who participated in this study.

\section{References}

1. Muduma G, Odeyemi I, Smith-Palmer J, et al. Review of the Clinical and Economic Burden of Antibody-Mediated Rejection in Renal Transplant Recipients. Adv Ther. 2016, 33(3):345-356.

2. Sellares $J$, de Freitas DG, Mengel $M$, et al. Understanding the causes of kidney transplant failure: the dominant role of antibody-mediated rejection and nonadherence. Am J Transplant 2012, 12(2):388399.

3. Wang JH, Skeans MA, Israni AK. Current Status of Kidney Transplant Outcomes: Dying to Survive. Adv Chronic Kidney Dis. 2016. 23(5):281-286.

4. Nicholson ML, Wheatley TJ, Horsburgh T, et al. The relative influence of delayed graft function and acute rejection on renal transplant survival. Transpl Int. 1996. 9(4):415-9.

5. Bloom RD, Bromberg JS, Poggio ED, et al. Cell-Free DNA and Active Rejection in Kidney Allografts. J Am Soc Nephrol. 2017. 28(7):2221-2232

6. Bouatou Y, Viglietti D, Pievani D,et al. Response to Treatment and Long-Term Outcomes in Kidney Transplant Recipients With Acute T Cell-Mediated Rejection. Am J Transplant. 2019. 19(7):19721988.

7. Li N, Li L, Chen Y. The Identification of Core Gene Expression Signature in Hepatocellular Carcinoma. Oxid Med Cell Longev. 2018. 2018:3478305. 
8. Zhang L, Yang Y, Cheng L, et al. Identification of Common Genes Refers to Colorectal Carcinogenesis with Paired Cancer and Noncancer Samples. Dis Markers. 2018. 2018:3452739.

9. Li L, Cai L, Zheng L, et al. Gefitinib Inhibits Bleomycin-Induced Pulmonary Fibrosis via Alleviating the Oxidative Damage in Mice. Oxid Med Cell Longev. 2018. 2018:8249693.

10. Li L, Zhao H, Chen B, et al. Noninvasive Identification of Immune-Related Biomarkers in Hepatocellular Carcinoma. J Oncol. 2019. 2019:2531932.

11. Racusen L, Rayner D, Trpkov K, et al. The Banff classification of renal allograft pathology: where do we go from here? Transplant Proc. 1996. 28(1):486-488.

12. Liu M, Xu Z, Du Z, et al. The Identification of Key Genes and Pathways in Glioma by Bioinformatics Analysis. J Immunol Res. 2017. 2017:1278081.

13. Li L, Lei Q, Zhang S, et al. Screening and identification of key biomarkers in hepatocellular carcinoma: Evidence from bioinformatic analysis. Oncol Rep. 2017. 38(5):2607-2618.

14. Gaudet P, Škunca N, Hu JC, et al. Primer on the Gene Ontology. Methods Mol Biol.2017. 1446:25-37.

15. Kanehisa M, Furumichi M, Tanabe M, et al. KEGG: new perspectives on genomes, pathways, diseases and drugs. Nucleic Acids Res. 2017. 45(D1): D353-D361.

16. Huang DW, Sherman BT, Tan Q, et al. DAVID Bioinformatics Resources: expanded annotation database and novel algorithms to better extract biology from large gene lists. Nucleic Acids Res. 2007. 35(Web Server issue): W169-75.

17. Feng H, Gu ZY, Li Q, et al. Identification of Significant Genes with Poor Prognosis in Ovarian Cancer via Bioinformatical Analysis. J Ovarian Res. 2019. 12(1):35.

18. Vikas R Dharnidharka, Kenneth E Lamb, Jie Zheng, et al. Lack of Significant Improvements in LongTerm Allograft Survival in Pediatric Solid Organ Transplantation: A US National Registry Analysis. Pediatr Transplant. 2015. 19(5):477-483.

19. A Hart, J M Smith, M A Skeans, et al. OPTN/SRTR 2015 Annual Data Report: Kidney. Am J Transplant. 2017. Suppl 1(Suppl 1):21-116.

20. Vikas R Dharnidharka, Andrew Malone. Biomarkers to Detect Rejection After Kidney Transplantation. Pediatr Nephrol. 2018. 33(7):1113-1122.

21. Silke Roedder, Tara Sigdel, Nathan Salomonis, et al. The kSORT Assay to Detect Renal Transplant Patients at High Risk for Acute Rejection: Results of the Multicenter AART Study. PLoS Med. 2014. 11(11):e1001759.

22. Valentin Haug, Branislav Kollar, Doha Obed, et al. The Evolving Clinical Presentation of Acute Rejection in Facial Transplantation. JAMA Facial Plast Surg. 2019. 21(4):278-285.

23. Scott Davis, James E Cooper. Acute Antibody-Mediated Rejection in Kidney Transplant Recipients. Transplant Rev (Orlando). 2017. 31(1):47-54.

24. Susan S Wan, Tracey D Ying, Kate Wyburn, et al. The Treatment of Antibody-Mediated Rejection in Kidney Transplantation: An Updated Systematic Review and Meta-Analysis. Transplantation. 2018. 102(4):557-568. 
25. B J Orandi, E H K Chow, A Hsu, et al. Quantifying Renal Allograft Loss Following Early AntibodyMediated Rejection. Am J Transplant. 2015. 15(2):489-498.

26. A Bentall, L D Cornell, J M Gloor, et al. Five-year Outcomes in Living Donor Kidney Transplants With a Positive Crossmatch. Am J Transplant. 2013. 13(1):76-85.

27. Sofia Christakoudi, Manohursingh Runglall, Paula Mobillo, et al. Development of a Multivariable Gene-Expression Signature Targeting T-cell-mediated Rejection in Peripheral Blood of Kidney Transplant Recipients Validated in Cross-Sectional and Longitudinal Samples. EBioMedicine. 2019. 41:571-583.

28. Lena Schiffer, Flavia Wiehler, Jan Hinrich Bräsen, et al. Chemokine CXCL13 as a New Systemic Biomarker for B-Cell Involvement in Acute T Cell-Mediated Kidney Allograft Rejection. Int J Mol Sci. 2019. 20(10):2552.

29. M D Parkes, P F Halloran, L G Hidalgo. Mechanistic Sharing Between NK Cells in ABMR and Effector T Cells in TCMR. Am J Transplant. 2018. 18(1):63-73.

30. Romney S Haylett, Norbert Koch, Lothar Rink. MHC class II molecules activate NFAT and the ERK group of MAPK through distinct signaling pathways in B cells. Eur J Immunol. 2009;39(7):19471955.

31. Soo-Ho Choi, Ayelet Gonen, Cody J Diehl, et al. SYK Regulates Macrophage MHC-II Expression via Activation of Autophagy in Response to Oxidized LDL. Autophagy. 2015;11(5):785-795.

32. Murat Kürtüncü, Vuslat Yılmaz, Halil ibrahim Akçay, et al. Impact of Fingolimod on CD4+ T Cell Subset and Cytokine Profile of Relapsing Remitting Multiple Sclerosis Patients. J Neuroimmunol. 2019. 337:577065.

33. Nicole L Sullivan, Christopher S Eickhoff, Xiuli Zhang, et al. Importance of the CCR5-CCL5 Axis for Mucosal Trypanosoma Cruzi Protection and B Cell Activation. J Immunol. 2011. 187(3):1358-1368.

34. J-H Li, B Zhao, X-H Zhu, et al. Blockade of Extracellular HMGB1 Suppresses Xenoreactive B Cell Responses and Delays Acute Vascular Xenogeneic Rejection. Am J Transplant. 2015. 15(8):20622074.

35. M D Parkes, P F Halloran, L G Hidalgo. Mechanistic Sharing Between NK Cells in ABMR and Effector T Cells in TCMR. Am J Transplant. 2018. 18(1):63-73.

36. K Sabapathy, T Kallunki, J P Davi, et al. c-Jun NH2-terminal Kinase (JNK)1 and JNK2 Have Similar and Stage-Dependent Roles in Regulating T Cell Apoptosis and Proliferation. J Exp Med. 2001. 193(3):317-328.

37. Triparna Sen, B Leticia Rodriguez, Limo Chen, et al. Targeting DNA Damage Response Promotes Antitumor Immunity Through STING-Mediated T-cell Activation in Small Cell Lung Cancer. Cancer Discov. 2019. 9(5):646-661.

38. Michael T Lotze, Kevin J Tracey. High-mobility Group Box 1 Protein (HMGB1): Nuclear Weapon in the Immune Arsenal. Nat Rev Immunol. 2005. 5(4):331-342.

39. Danila Vella, Simone Marini, Francesca Vitali, et al. MTGO: PPI Network Analysis Via Topological and Functional Module Identification. Sci Rep. 2018. 8(1):5499. 
40. Dany Anglicheau, Vijay K Sharma, Ruchuang Ding, et al. MicroRNA Expression Profiles Predictive of Human Renal Allograft Status. Proc Natl Acad Sci U S A. 2009. 106(13):5330-5335.

41. Ingo Büssing, Jr-Shiuan Yang, Eric C Lai, et al. The Nuclear Export Receptor XPO-1 Supports Primary miRNA Processing in C. Elegans and Drosophila. EMBO J. 2010. 29(11):1830-1839.

42. Gur P Kaushal, Kiran Chandrashekar, Luis A Juncos. Molecular Interactions Between Reactive Oxygen Species and Autophagy in Kidney Disease. Int J Mol Sci. 2019. 20(15):3791.

43. Michael H Yan, Xinglong Wang, Xiongwei Zhu. Mitochondrial Defects and Oxidative Stress in Alzheimer Disease and Parkinson Disease. Free Radic Biol Med. 2013. 62:90-101.

44. K Sennvik, J Fastbom, M Blomberg, et al. Levels of Alpha- And Beta-Secretase Cleaved Amyloid Precursor Protein in the Cerebrospinal Fluid of Alzheimer's Disease Patients. Neurosci Lett. 2000. 278(3):169-172.

\section{Figures}

\section{Figure 1}

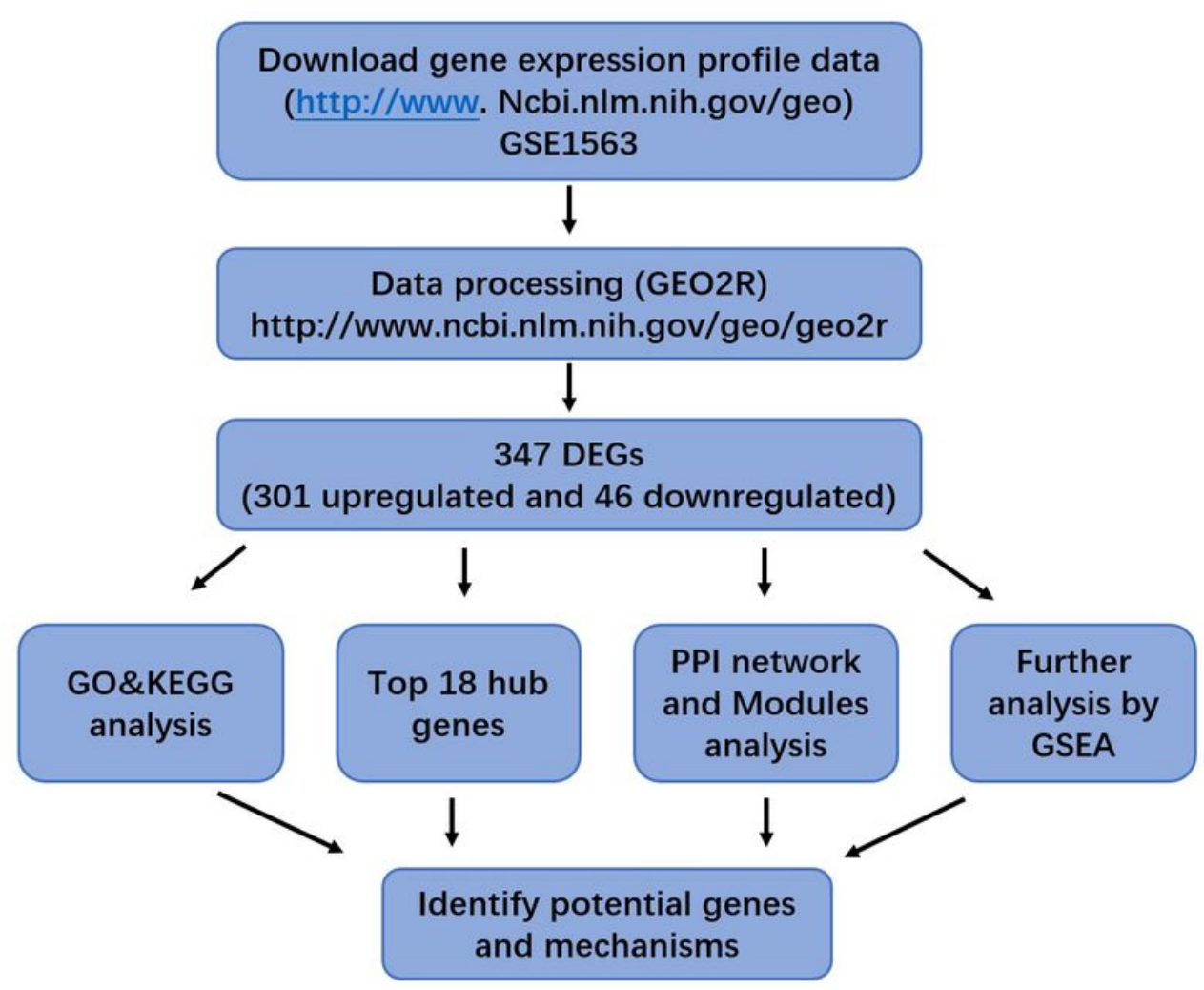

\section{Figure 1}

Flow diagram of the analysis process data collection, preprocessing, analysis and verification. 
Figure 2

A

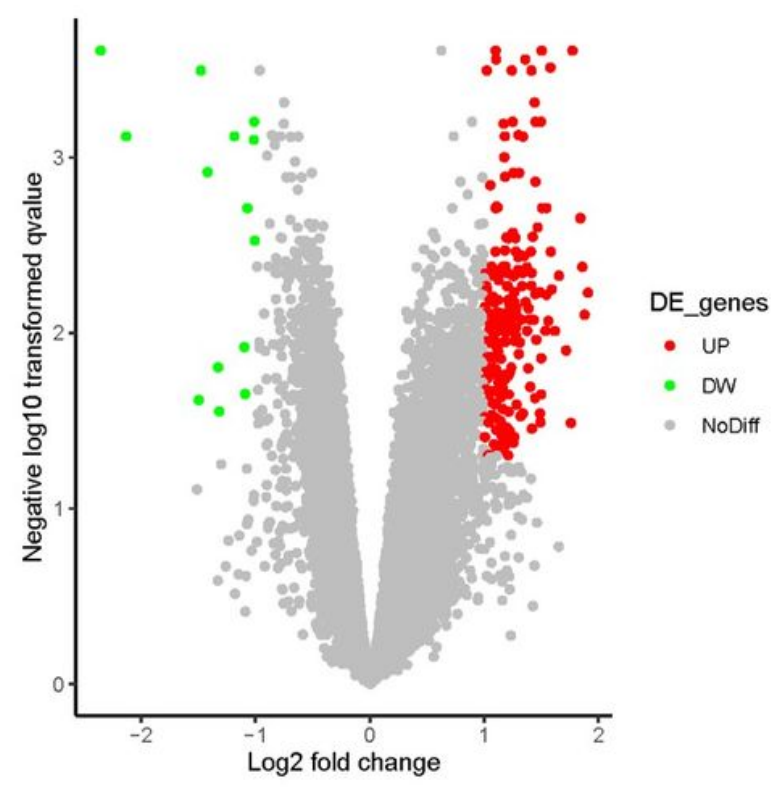

B

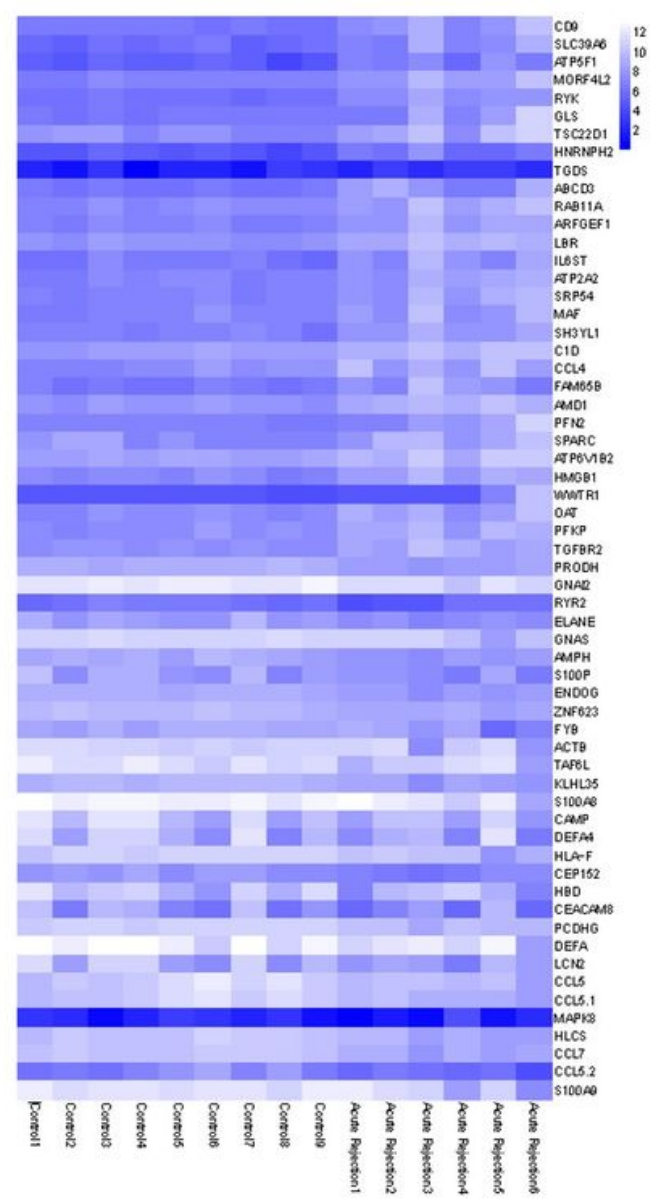

Figure 2

Identification of DEG of PBL in GSE1563. (A) Volcano plot of total 347 DEGs detected in AR from GSE1563. The red dots are on behalf of 301 upregulated DEGs, while green dots are on behalf of 46 downregulated DEGs. The gray dots mean the no differentially expressed genes. (B) Heatmeap of the expression levels of the top 30 upregulated DEGs and top30 downregualted DEGs applying the GEO2R online analysis tool on the basis of $\log \mathrm{FC} \leq-1$ or $\log \mathrm{FC} \geq 1$ and $\mathrm{P}$ value $<0.05$ criteria. 
Figure 3

A

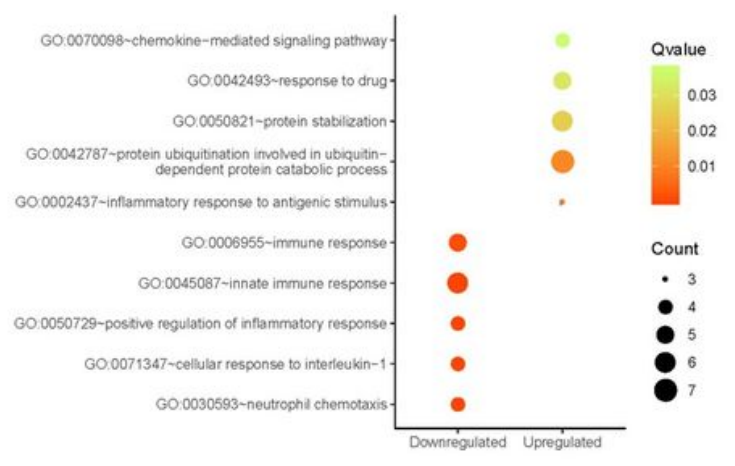

C

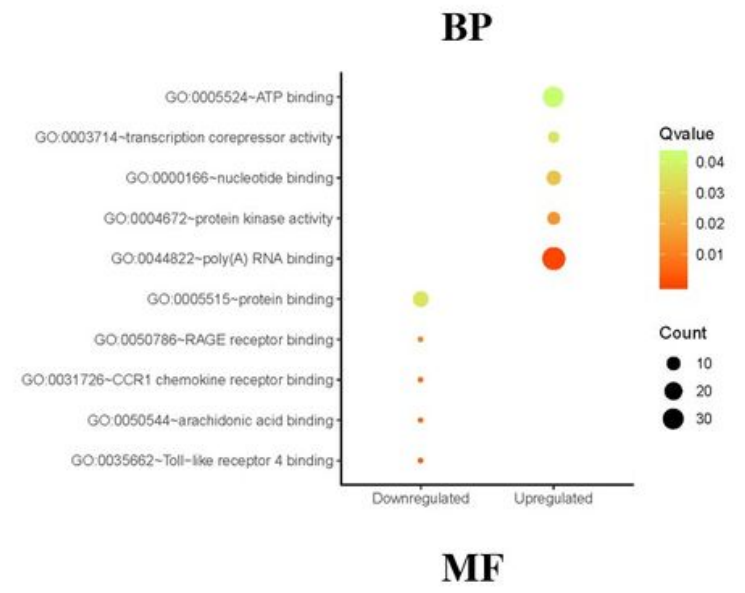

B

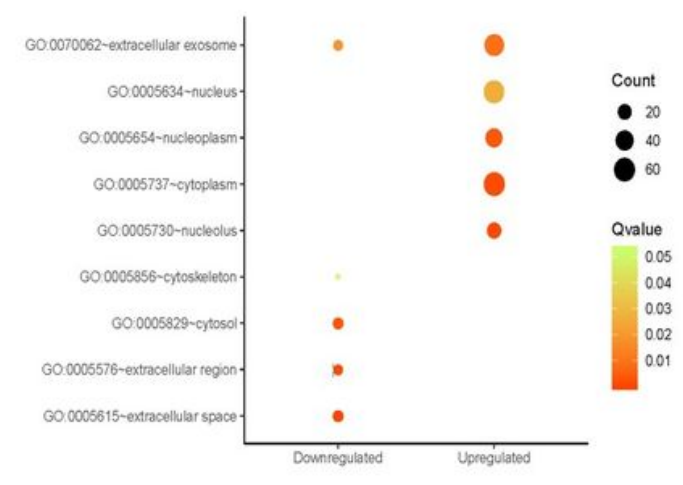

D

CC

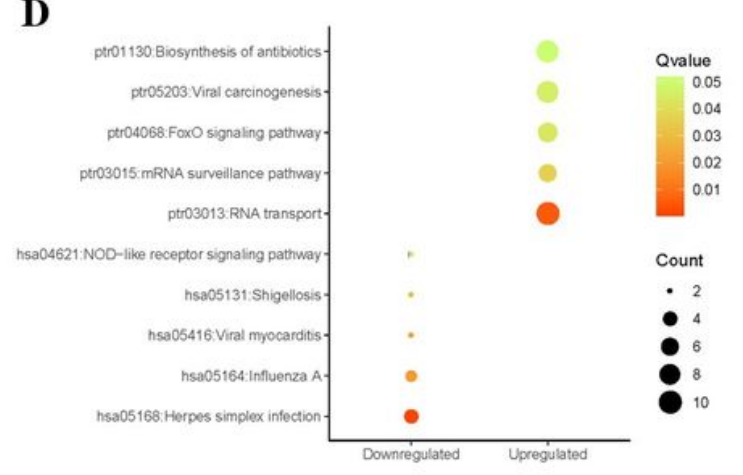

KEGG

\section{Figure 3}

The scatter plots of GO and KEGG pathway enrichment plot of AR. (A) The GO pathway enrichment scatter plot in the biological process (BP);(B) The GO pathway enrichment scatter plot in the cellular component (CC); (C) The GO pathway enrichment scatter plot in the molecular function (MF); (D) KEGG pathway enrichment scatter plots of AR. 
Figure 4

A

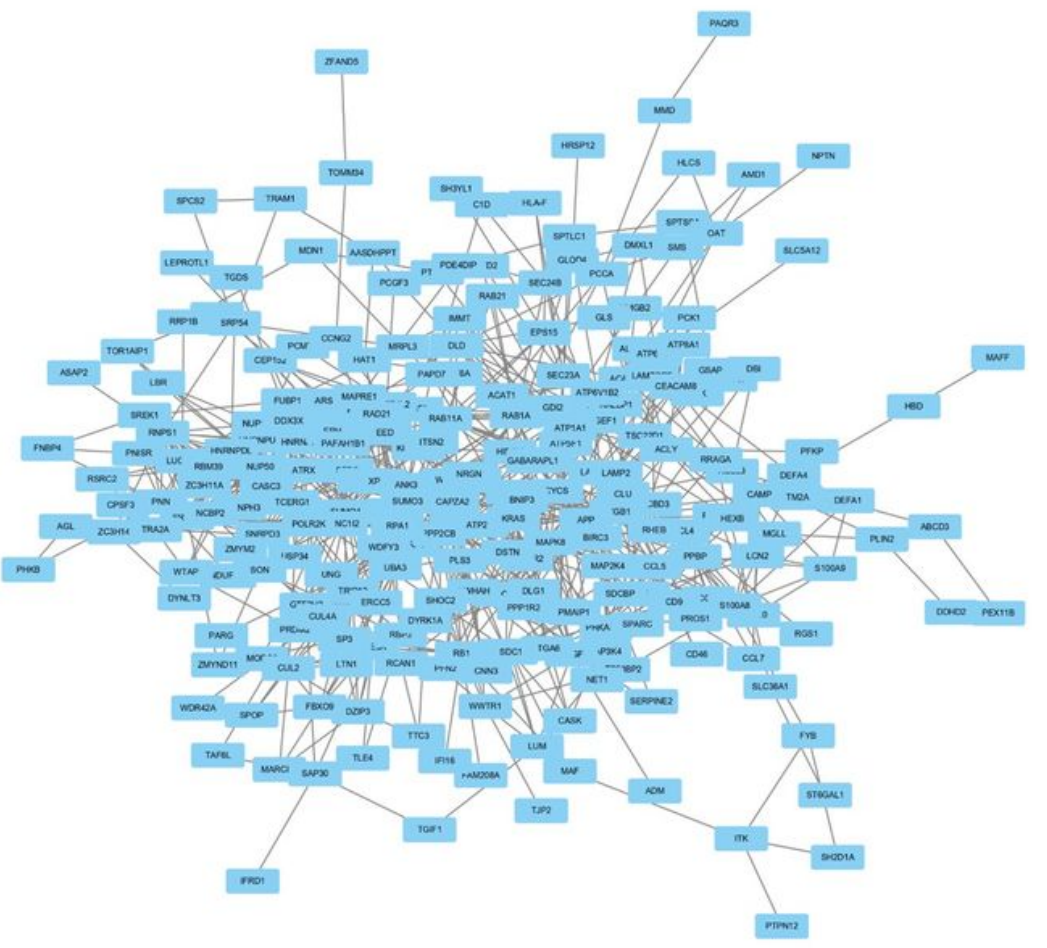

B

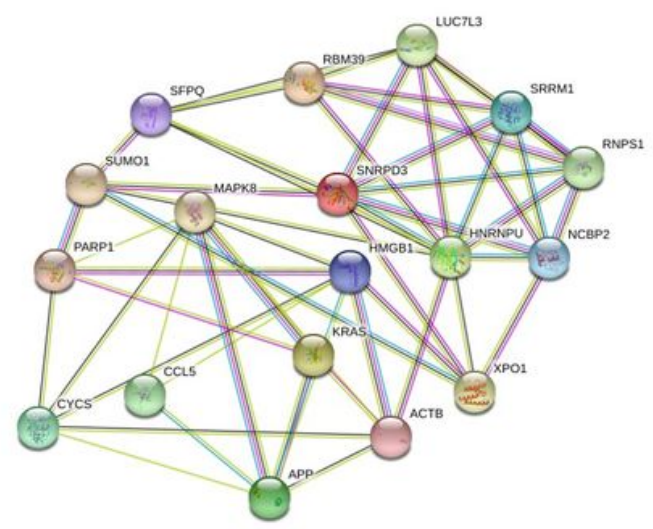

\section{Figure 4}

Protein-protein interaction(PPI) network. (A) The PPI network of entire DEGs. (B) The PPI network of top 18 hub genes with high connectivity degree according to the information of the STRING protein query with maximum number of interactors $\leq 5$, confidence score $\geq 0.4$.

\section{Figure 5}

A

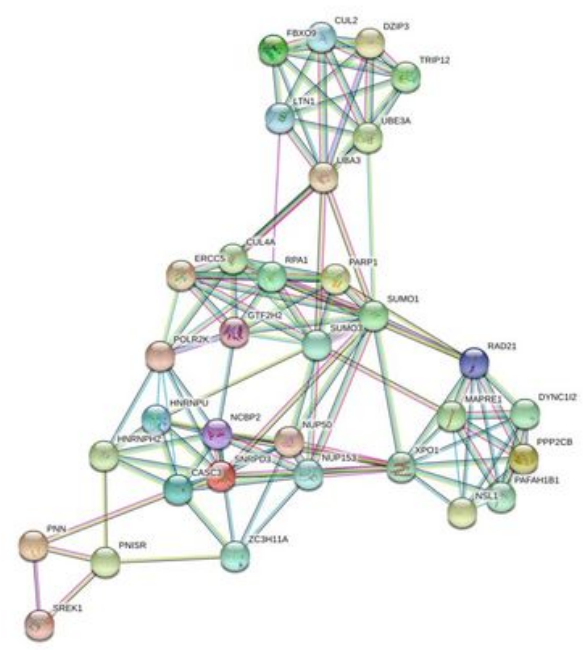

B

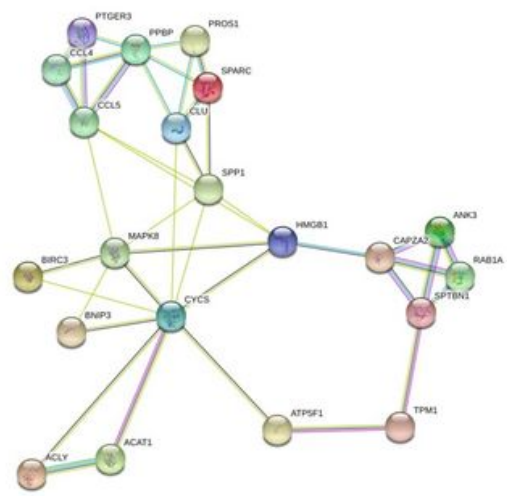

C

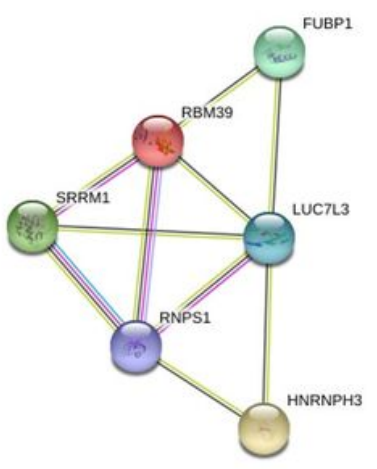

Figure 5 
The module analysis of AR (A) The PPI network of Module 1 (B) The PPI network of Module 2 (C) The PPI network of Module 3.

\section{Figure 6}

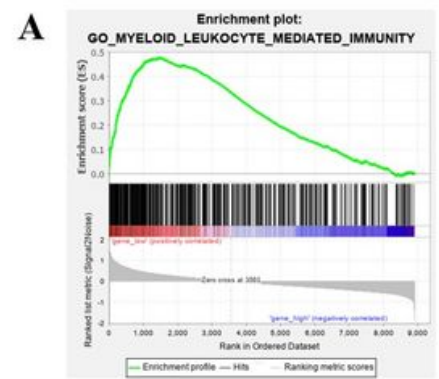

MAPK8

Enrichment score(ES):0.48

Nominal P-value: $<0.01$

FDR q-value: $<0.01$

D

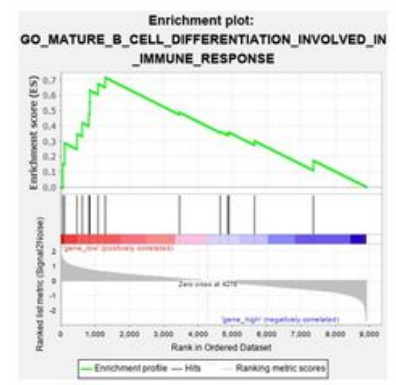

HMGB1

Enrichment score(ES):0.71

Nominal P-value: $<0.01$

FDR q-value: $<0.01$

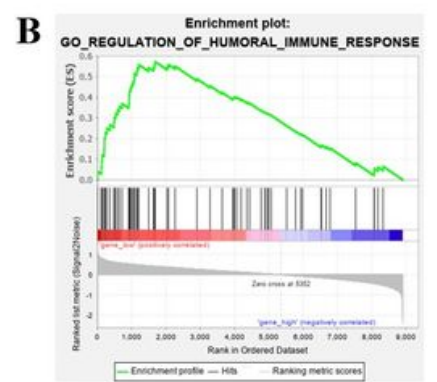

CCL5

Enrichment score(ES):2.31

Nominal P-value: $<0.01$

FDR q-value: $<0.01$

E

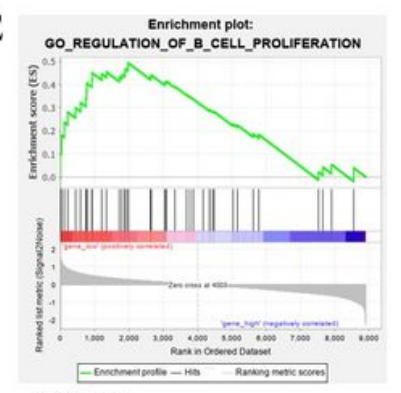

NCBP2

Enrichment score(ES):1.93

Nominal P-value $:<0.01$

FDR q-value:0.10
C

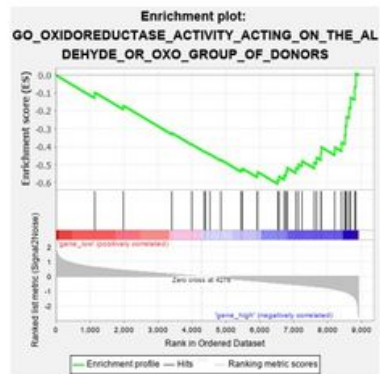

APP

Enrichment score(ES):-2.15

Nominal P-value: $<0.01$

FDR q-value:0.01

F Enrichment plot: OO_NCRNA_EXPORT_FROM_NUCLEUS

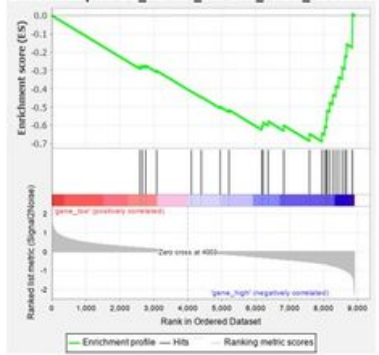

XPO1

Enrichment score(ES):-0.69

Nominal P-value: $<0.01$

FDR q-value:0.02

\section{Figure 6}

Gene set enrichment analysis of AR (A) MAPK8 is negatively correlated with myeloid leukocyte mediated immunity; (B) CCL5 is negatively correlated with regulation of humoral immune response; (C) APP is positively correlated with oxidoreductase activity acting on the aldehyde or oxo group of donors; (D) HMGB1 is negatively correlated with mature $B$ cell differentiation involved in immune response; $(E)$ NCBP2 is negatively correlated with regulation of B cell proliferation; $(F)$ XPO1 is positively correlated with ncRNA export from nucleus" 